\title{
Correction to: An empirical constitutive model for complex glass-forming liquids using bitumen as a model material
}

\author{
Olli-Ville Laukkanen ${ }^{1,2} \cdot$ H. Henning Winter ${ }^{1} \cdot$ Hilde Soenen $^{3} \cdot$ Jukka Seppälä $^{2}$
}

Published online: 9 January 2018

(C) Springer-Verlag GmbH Germany, part of Springer Nature 2018

\section{Correction to: Rheologica Acta}

https://doi.org/10.1007/s00397-017-1056-6

The original version of this article unfortunately contained a mistake.

In Eq. (9), the words 'sin' and 'with' should not be written in italics. Please find below the correct Eq. 9:

$\begin{aligned} H(\tau) & =-\frac{G_{g}}{\pi u} \sum_{k=1}^{\infty} \frac{(-1)^{k}}{k !} \sin \left(\pi \beta_{K W W} k\right) \Gamma\left(\beta_{K W W} k+1\right) u^{\left(\beta_{K W W} k+1\right)}, \\ \text { with } u & =\frac{\tau}{\tau_{K W W}}\end{aligned}$

Equation (12) represents the C-D model, not the C-C model. This typing error is not present in the revised manuscript that was submitted to the journal on November 10, but has been introduced during the typesetting process. Also, the commas have been placed incorrectly; they should be located at the end of the equations (before the word 'for'), not at the end of the whole row. We have pointed this out during the typesetting process. Please find below the correct Eq. 12:

$$
\begin{aligned}
& H(\tau)=G_{g} \frac{\sin \beta \pi}{\pi}\left(\frac{\tau}{\tau_{0}-\tau}\right)^{\beta}, \text { for } \tau<\tau_{0} \\
& H(\tau)=0, \text { for } \tau>\tau_{0}
\end{aligned}
$$

The original article has been corrected.

The online version of the original article can be found at https://doi.org/ 10.1007/s00397-017-1056-6

Olli-Ville Laukkanen

olaukkanen@mail.pse.umass.edu

1 Department of Polymer Science and Engineering and Department of Chemical Engineering, University of Massachusetts,

Amherst, MA 01003, USA

2 Department of Chemical and Metallurgical Engineering, School of Chemical Engineering, Aalto University, P.O. Box 16100, 00076 Aalto, Finland

3 Nynas NV, 2000 Antwerp, Belgium 